\title{
1 A Shear Wave Ground Surface Vibration Technique for the Detection of Buried Pipes
}

3

J M Muggleton* and B. Papandreou

Institute of Sound and Vibration Research, University of Southampton, Southampton, UK, SO17 1BJ

*Corresponding author, e-mail: jmm @ isvr.soton.ac.uk

\section{Abstract}

A major UK initiative, entitled 'Mapping the Underworld' aims to develop and prove the efficacy of a multi-sensor device for accurate remote buried utility service detection, location and, where possible, identification. One of the technologies to be incorporated in the device is low-frequency vibro-acoustics; the application of this technology for detecting buried infrastructure, in particular pipes, is currently being investigated. Here, a shear wave ground vibration technique for detecting buried pipes is described. For this technique, shear waves are generated at the ground surface, and the resulting ground surface vibrations measured. Time-extended signals are employed to generate the illuminating wave. Generalized crosscorrelation functions between the measured ground velocities and a reference measurement adjacent to the excitation are calculated and summed using a stacking method to generate a cross-sectional image of the ground. To mitigate the effects of other potential sources of vibration in the vicinity, the excitation signal can be used as an additional reference when calculating the cross-correlation functions. Measurements have been made at two live test sites to detect a range of buried pipes. Successful detection of the pipes was achieved, with the use of the additional reference signal proving beneficial in the noisier of the two environments. 


\section{I. INTRODUCTION}

2 There is a requirement to be able to detect object buried at a shallow depth, typically $1-2 \mathrm{~m}$, in

3 the ground. Typical target objects of interest include buried infrastructure (Royal et al., 2011),

4 such as water and gas pipes, archaeological artefacts and buried ordnance. The problems

5 associated with inaccurate location of buried pipes and cables have been serious for many

6 years and are getting worse as a result of increasing traffic congestion in the UK's major

7 urban areas. The problems primarily derive from the fact that the vast majority of the buried

8 utility infrastructure exists beneath roads and therefore any excavation is likely to disrupt the

9 traffic. A recent UK study estimated that streetworks cost the UK £7bn annually; comprising

$10 £ 5.5 \mathrm{bn}$ in social and indirect costs and $£ 1.5 \mathrm{bn}$ in direct costs (McMahon et. al., 2005). The

11 location techniques that are currently commercially available are either simple (yet strictly

12 limited in their ability to detect the wide variety of utilities) and carried out immediately prior

13 to excavation by site operatives or are more sophisticated and carried out by specialist

14 contractors. Controlled trials carried out by UK Water Industry Research have shown that,

15 even when sophisticated detection techniques are employed, detection rates are often poor

16 (Ashdown, 2000) and, as a result, far more excavations are carried out than would otherwise

17 be necessary for maintenance and repair. While a variety of techniques using different

18 technologies are available, all suffer from the same essential drawback that, when deployed

19 alone, they will not provide an adequate solution to the problem; moreover, all have their own

20 specific limitations.

21 Much of the technological focus on the detection of such objects utilises electromagnetic

22 phenomena (Linford, 2006). These technologies have limited ability to detect non-metallic or

23 interfacing with weakly contrasting permittivity/permeability. In addition, those technologies

24 featuring electromagnetic wave propagation, such as ground penetrating radar and metal

25 detectors, suffer from high attenuation in wet or conductive media, resulting in limitations on

26 their performance. Currently a large multi-centre programme, Mapping the Underworld (see

27 www.mappingtheunderworld.ac.uk ), is being undertaken in the UK to assess the feasibility 
1 of a range of potential technologies that can be combined into a single device to accurately

2 locate buried pipes and cables. The potential technologies include ground penetrating radar,

3 low-frequency quasi-static electromagnetic fields, passive magnetic fields and low frequency

4 vibro-acoustics and significant advances have already been made (Royal et al, 2006,2011).

5 Vibro-acoustic techniques offer performance in media in which electromagnetic alternatives

6 struggle and, furthermore, have the potential to image differences in mechanical, rather than

7 electromagnetic, impedance. Vibro-acoustic techniques are thus worthy of development as

8 they have the potential to complement the more mature existing imaging technologies.

9 Previous work (Muggleton et al, 2008,2011) addressed the detection of plastic water pipes in 10 situations where access to some part of the pipe could be gained at the ground surface. 11 Although successful, the access requirement limits the wider potential of this technique. A prior feasibility study, however (Muggleton et al, 2006), also identified a second deployment

13 strategy - direct excitation of the ground. When the ground is mechanically excited, waves 14 will propagate away from the excitation point. Depending on the form of excitation, different 15 wave types will be excited in the ground and these waves can be detected, depending of 16 course on the frequency. Furthermore, they will be scattered by objects, such as pipes and cables, whose mechanical properties are different from the soil, even if they are buried several metres deep, provided that the object is larger than about half a wavelength. Detection and 19 analysis of the scattered waves, in principle, allows the pipes/cables to be accurately located.

20 Acoustic imaging in the ground is historically associated with hydrocarbon exploration. This 21 application has been successful in deep imaging of geological layers to reveal the location of 22 hydrocarbon deposits. The imaging methods are usually based on the common depth point 23 (CDP) stack technique (Schnieder, 1984), which employs time domain stacking. An 24 impulsive source is used along with multiple sensors whose time histories are summed, after 25 accounting for propagation delays, to form an image. However, these methods cannot be 26 directly scaled to shallow depths due to a variety of problems. These include the much shorter 27 separation time between the waves propagating directly from sources to sensors over the 
1 ground's surface and those reflected from buried surfaces, the requirement for higher

2 frequencies, and the possibility of substantial near surface variations in wavespeeds (Miller

3 and Xia, 1988).

4 The detection of objects buried at a shallow depth has had comparatively little attention.

5 Notable attempts to detect buried objects at very shallow depths $(<10 \mathrm{~cm})$ using Rayleigh

6 waves have been made, chiefly with the aim of landmine detection (Sabatier and Gilbert,

7 2000, Scott et al, 2001). Whilst these methods are capable of achieving good results for

8 targets just under the surface of the ground they are not applicable to the slightly more deeply

9 buried targets of interest in this paper because of the rapid attenuation of the Rayleigh wave

10 with depth. However, Sugimoto et al (2000) have adapted the time domain stacking technique

11 for the detection of objects buried at more shallow depths. More recently, the signal stacking

12 method has been further modified by using time extended signals, an alternative excitation

13 method and additional signal processing techniques (Papandreou, 2011, Papandreou et al,

$142009,2011)$ and has been used with some success to detect concrete pipes and slabs. It is this

15 technique which is adapted here and employed with the aim of detecting a range of buried

16 pipes.

17 At this stage the work sits firmly in the research arena. The authors do not claim that

18 the technique in its present form could be integrated into a device which could be

19 deployed successfully in an urban environment; rather, it is our intention to

20 demonstrate its potential and reveal some of the underlying physical processes in

21 play.The paper is organised as follows: section 2 gives a description of the fundamentals of

22 the experimental method, the signal processing adopted along with an enhancement for use in

23 noisy environments; in section 3 the imaging experiments are described and the results

24 presented; finally, section 4 presents the conclusions and makes recommendations for further

25 work. 


\section{II. DESCRIPTION OF THE METHOD}

\section{A Fundamentals}

3 The basic experimental method used in this publication has been previously documented

4 (Papandreou, 2011, Papandreou et al, 2011), and consequently only a brief synopsis is

5 provided here. It is an active method that produces a cross-sectional two-dimensional image

6 through the ground. A seismic source produces a time-extended signal to illuminate the

7 buried object. Reflections are then measured using an array of surface sensors. The

8 experimental setup is shown in Figure 1.

9 To form an image the distances from the source to sensor locations via each pixel on the 10 image plane are first calculated. These are related to times of flight by the speed of the wave 11 which is assumed to reflect from the target and propagate to the sensors. Due to high 12 variability of the wavespeed between locations and with soil conditions an estimate of the 13 wavespeed is required.

14 If an impulsive source has been used (e.g. hammer strike) then the measured time histories 15 will contain a peak at the time of flight of a reflected signal. Thus by making the pixel value 16 equal to the amplitude of the measured signal at this time, the reflection is mapped from the 17 time domain to the image plane, with each peak in the time domain generating an elliptical 18 curve. Repetition of this procedure for each of the geophones and summation of the resultant 19 images will, provided that the wavespeed has been accurately estimated, produce a peak in 20 the image that indicates the location of a target. The signal to noise ratio can be improved by 21 moving the source, repeating the method, and summing the resultant image. This also reduces the impact of anomalous dead-patches or regions of high heterogeneity.

23 As discussed previously, the method has been adapted by using time extended signals rather 24 than impulses. This enables both greater control of the frequency content of the input signal 25 and, moreover, the ability to input larger amount of energy without resorting to input 
1 amplitudes that produce a non-linear response in the ground. In order to incorporate time-

2 extended signals into the imaging method cross-correlations functions are used.

3 A form of the cross-correlation function useful for practical implementation is given by

$4 \quad$ Bendat and Piersol (1993)

$$
R_{x y}(\tau)=\int_{-\infty}^{\infty} S_{x y}(f) e^{2 \pi i f t} d f
$$

6 where $S_{x y}(f)$ is the cross-spectral density between a reference signal (provided by a

7 geophone located close to the source at $x$ ) and the $y^{\text {th }}$ measurement sensor and $f$ is frequency.

8 Equation (1) is a statement that the cross-correlation function is equal to the inverse Fourier

9 transform of the cross-spectral density. Numerical implementation of the cross-correlation

10 functions uses the relation of Equation (1) to exploit the computational efficiency of the fast

11 Fourier transform. This also allows for generalisations to the cross-correlation function that

12 are now discussed.

\section{B Smoothed Coherence Transform}

14 It is desirable to minimise the width of peaks in the cross-correlation functions prior to 15 application of the imaging algorithm. The reason for this is two-fold: firstly, if the cross16 correlation peaks are too wide then multiple peaks in the cross-correlation domain due, for 17 example, to the propagation of multiple wave types, may coalesce; secondly, if the peaks are 18 very wide, then a cross-correlation function with a single peak will produce an image where 19 all regions have a high value with only a relatively minor peak at the target location, making target detection more difficult.

21 Limitations on the bandwidth of time domain signals results in an increase in the width of 22 these peak (White, 1969). Due to the high attenuation of waves propagating in the ground 23 there are unavoidable bandwidth limitations in the measured data. The effects of this limited 24 bandwidth are reduced by the use of generalised cross-correlation functions (GCCs). These 
1 involve the incorporation of a frequency dependent weighting function (Knapp and Carter,

2 1976), $\psi(f)$, to Equation (1) to give

$$
R_{x y}(\tau)=\int_{-\infty}^{\infty} \psi(f) S_{x y}(f) e^{2 \pi i f t} d f
$$

4 There are several different weighting functions could be appropriate; these have been

5 discussed extensively in the context of leak detection by Gao et al (2006) and include the 6 phase transform (PHAT) and the smoothed coherence transform (SCOT). The PHAThas previously been used for the shear wave method (Papandreou et al, 2011) and is defined by

$$
\psi_{\text {PHAT }}(f)=\left|S_{x y}(f)\right|^{-1}
$$

9 The PHAT has the effect of 'pre-whitening' the signal by setting the magnitude of the crossspectral density to unity whilst preserving the phase information. This is effective because the

11 estimation of the time delay requires only knowledge of the phase, and the attenuated 12 magnitude information serves only to broaden the cross-correlation peaks. A drawback of the 13 PHAT is that it takes no account of the coherence of the signal and therefore increases the 14 magnitude of regions of the cross-spectral densities that correspond only to noise, reducing the accuracy of the time delay estimate.

16 An alternative weighting function is the smoothed coherence transform (SCOT) (Carter et al, 17 1973). This is similar to the PHAT, but in addition to pre-whitening, the cross-spectral density 18 is also weighted by the coherence function. The coherence function is an indication of the 19 strength of linear relation between two signals (Shin and Hammond, 2008). Frequency 20 regions of the received signal unrelated to the input thus have an appropriately reduced 21 contribution to the calculation of the cross-correlation function. Mathematically the weighting function is 


$$
\psi_{\text {SСOT }}(f)=\frac{\gamma_{x y}(f)}{\left|S_{x y}(f)\right|}
$$

2 where $\gamma_{x y}$ denotes the coherence function between the reference signal and the signal

3 measured at the $y^{\text {th }}$ geophone. The coherence function is defined as

$$
\gamma_{x y}{ }^{2}(f)=\frac{\left|S_{x y}(f)\right|^{2}}{S_{x x}(f) S_{y y}(f)}
$$
where $S_{x x}$ and $S_{y y}$ denote the auto-spectral density functions of the reference geophone and the

$6 y^{\text {th }}$ measurement geophone respectively (Shin and Hammond, 2008). Substitution of Equation

7 (4) into Equation (3) gives the form used for implementation:

$$
\psi_{\text {SCOT }}(f)=\frac{1}{\sqrt{S_{x x}(f) S_{y y}(f)}}
$$

9 Using the estimated wavespeed the value of the cross-correlation functions at times 10 corresponding to the calculated delays are summed at each pixel location to form an image. If 11 the image pixel corresponds to a reflector then the contribution from each cross-correlation 12 function should be larger and thus produce a peak in the image. If the image pixel does not 13 correspond to location of a reflector then, whilst the contribution from an individual crosscorrelation may be maximal, the combined contribution of all cross-correlation functions will not.

\section{Use of an additional reference for noisy environments}

17 One disadvantage of using the form of the cross-correlation, $R_{x y}(\tau)$, given in equation (2) is 18 that if there are any other proximate vibration sources, these will be picked up by both the 19 reference sensor and the measurement sensors and contaminate the measurements, giving rise 20 to time delays not corresponding to the correct source-target receiver distances. This can be 21 countered by using a slightly modified form of the cross-correlation, $R_{x y} V_{r e f}(\tau)$, in which the 
1 excitation signal is used as an additional reference so that only signals correlated with the

2 input signal will contribute to the result, viz.

$$
R_{x y V_{r e f}}(\tau)=\int_{-\infty}^{\infty} \psi_{x V}(f) \psi_{y V}(f) S_{x V}(f) S_{y V} *(f) e^{2 \pi i f t} d f
$$

4 where the subscript $V$ refers to the voltage input to the shaker, i.e. the excitation signal, and

5 the $*$ denotes the complex conjugate.

\section{III. IMAGING EXPERIMENTS}

\section{A Experimental setup}

8 Throughout this paper the source used is an inertial electromagnetic shaker, specifically a

9 Wilcoxon Research F4 shaker with its associated Wilcoxon power amplifier. The geophones were input/output SM-24's, which were attached directly into an eight channel Prosig P-8000 data acquisition unit.

12 For all the experiments, the ground was excited horizontally with an inertial shaker in order to 13 preferentially excite horizontally polarized shear waves; to give some directionality, 14 elongated contact with the ground was achieved using the inertial shaker attached to a rake. 15 The rake was placed in the ground so that the motion of the shaker was parallel to the pipe, thus producing shear waves travelling across it. The rake assembly is shown in Figure 2.

17 Papandreou (2011) (chapter 2, pg 63) has shown previously that horizontal excitation can 18 produce more than $80 \%$ horizontally polarised shear waves with only $5 \%$ and $1 \%$ of the 19 energy exciting compressional and Rayleigh waves respectively. Conversely, the generation 20 of compressional waves inherently leads to the production of Rayleigh surfaces waves. The 21 propagation of additional wavetypes leads to a reduction in the quality of the image and the possibility of the reflected peak of interest being obscured in the cross-correlation functions.

23 Moreover, in using shear waves rather than compressional waves as previously (Papandreou 24 et al, 2009, 2011), their relatively low wavespeed increases the time separation between a 
1 reflected wave and a wave that is transmitted directly between the source and geophone. This

2 argument has also been detailed in Papandreou (2011) (chapter 3, page 79) using geometry

3 and wavespeed relations.

\section{B Experiments to locate a buried plastic water pipe}

5 The test site used for these experiments was a dedicated leakage research test site at Blithfield

6 Reservoir, Staffordshire, owned by South Staffs water. The Blithfield test site comprises a

7 single, 120m-long, 180mm-diameter, pressurized polyethylene mains water pipe, buried at the

8 standard burial depth of approximately $1 \mathrm{~m}$. It has several access points along its length

9 allowing for the simulation of leaks.

10 The shaker and rake assembly was positioned at each geophone location in turn, thus enabling

11 the stacking to be performed over $49(7 \times 7)$ source-receiver position combinations. The 12 excitation signal was a swept sine from $10 \mathrm{~Hz}-400 \mathrm{~Hz}$, over approximately 2 minutes, using a $131000 \mathrm{~Hz}$ sampling rate.

14 Measurements were made along three lines on the ground surface traversing the pipe at right15 angles, each line being $3 \mathrm{~m}$ apart. For each measurement line, seven geophones (type I-O SM16 24) were used, spaced at $1 \mathrm{~m}$ intervals. Seven excitation positions were used, thus enabling the stacking to be performed over $49(7 \times$ x $)$ source-receiver position combinations. The excitation signal was a swept sine from $10 \mathrm{~Hz}-400 \mathrm{~Hz}$, over approximately 2 minutes, using a

$191000 \mathrm{~Hz}$ sampling rate. Figure 3 shows one geophone line traversing the run of the pipe along 20 with the excitation platform.

21 That predominantly shear waves were being produced with the intended polarization can be verified with reference to Figure 4, which shows example velocity time histories in three orthogonal directions at the source, in this case at location 1 (Figure $4 \mathrm{a}$ ) and at the furthest receiver geophone, in this case location 7,6m distant (Figure $4 \mathrm{~b}$ ). Figure $4 \mathrm{a}$ shows that the ground is being excited predominantly in the intended direction, i.e. perpendicular to the measurement line, with the measured signal in this direction being approximately five times 
1 those in the other two directions. Figure $4 \mathrm{~b}$ reveals that, whilst not only horizontally polarized

2 shear waves in the intended direction are being generated and propagating, the magnitude of 3 the vertical velocity and horizontal velocity parallel to the measurement line are 4 approximately ten and five times smaller respectively, indicating that the intended shear 5 waves do dominate.

6 Stacking requires that the shear wave velocity through the ground in situ is known. This can 7 be calculated from the time delay associated with the largest peaks in the generalized 8 correlation function at different source-receiver positions although this method is not always 9 successful and can lead to unexpected and implausible results (Papandreou, 2011). The 10 reasons for this are unclear but may be associated with other wavetypes present in the ground.

11 Furthermore, different pairs of positions will give slightly different results so there is always 12 some uncertainty in the final wavespeed (which, of course, may vary slightly from location to 13 location). An alternative method is to examine the unwrapped phase of the modified cross14 spectrum between different source receiver pairs, as given by the integrand of Equation (3) 15 and then using the gradient method described previously (Muggleton et al, 2011).

16 For each dataset, the time delays associated with the largest two peaks in the correlation 17 functions were used to attempt to estimate the shear wavespeed as were the unwrapped phase 18 gradients. Whichever method was used, different pairings resulted in values ranging between 19 approximately $65 \mathrm{~m} / \mathrm{s}$ and $90 \mathrm{~m} / \mathrm{s}$. With this in mind, a range of speeds was used in performing 20 the stacking and obtaining the final cross-sectional images.

21 Figure 5 shows the cross-sectional images, obtained for six different wavespeeds $(65,70,75$, 2280,85 , and $90 \mathrm{~m} / \mathrm{s}$ ), along with the geophone positions on the surface and the estimated 23 location of the pipe (black circle - obtained from burial records) for the first measurement 24 line. The images have been presented with a colourmap such that areas of high intensity 25 (greatest reflectivity) are represented by dark red, through to areas of low intensity (least 26 reflectivity) being represented by blue. A colour bar is shown alongside the images. Thus 
1 dark red areas correspond to locations where the detection method has located a buried object

2 (in this case the pipe) A dark area can be seen in all the plots indicating the detection

3 locations. That these dark areas are in the vicinity of the black circle in each case suggests

4 that the pipe has indeed been detected by this method.

5 Examining the figures as a whole it can be seen that the perceived depth of the target

6 increases with the estimated wavespeed, varying from approximately $0.8 \mathrm{~m}$ at $65 \mathrm{~m} / \mathrm{s}$ to $1.7 \mathrm{~m}$

7 at $90 \mathrm{~m} / \mathrm{s}$. That the perceived depth increases with wavespeed is as expected given that

8 increasing the wavespeed in the stacking calculations results in increased source-target-

9 receiver propagation distances for the same time delays. For the three largest wavespeeds

$10(80 \mathrm{~m} / \mathrm{s}, 85 \mathrm{~m} / \mathrm{s}$ and $90 \mathrm{~m} / \mathrm{s})$, dark areas corresponding to the surface wave can be seen at depths

11 from $0.5 \mathrm{~m}$ to $1.0 \mathrm{~m}$, indicating that these wavespeeds are, indeed, too high. These dark areas

12 recede to the surface for the lowest three wavespeeds suggesting that these wavespeeds are

13 more appropriate. Using a wavespeed of $70 \mathrm{~m} / \mathrm{s}$ gives the best depth agreement with the burial

14 records, although laterally there is some discrepancy. The precise pipe burial location relative

15 to the geophone positions was not, however, confirmed by other means at the time of testing.

16 For the second measurement line (Figure 6, here showing two wavespeeds), dark areas in the 17 images again revealed the pipe location; here, however, the optimum wavespeed was found to 18 be between $80 \mathrm{~m} / \mathrm{s}$ and $85 \mathrm{~m} / \mathrm{s}$. For the third measurement line, no dark area in the image 19 corresponding to the pipe location could be satisfactorily identified.

20 For all three sets of measurements, no difference in the results was observed between using 21 the form of cross-correlation given in equation (2) and that including the shaker input voltage 22 as an additional reference as given in equation (7). This is as anticipated given that the 23 Blithfield site was an extremely quiet environment in which to make measurements.

24 In contrast, the effect of using the smoothed coherence transform is clearly evident. Figure 7 25 shows the cross-sectional images corresponding to those in figure 5, without performing the 26 pre-whitening using the SCOT. What is evident here is that, although a clear dark area can be 
1 seen in each plot, the resolution achieved from the contributions of higher frequencies is

2 absent. This can be seen more clearly with reference to figure 8. Figure 8a (note the log scale)

3 shows the magnitude of the integrand of equation (7) with the weighting functions, $\psi$, set to

4 unity, for an example source/receiver pairing. It can be seen that, without pre-whitening, only

5 frequencies around $90 \mathrm{~Hz}$ will contribute to the integrand as the magnitude tails off extremely

6 rapidly away from this frequency. Figure $8 b$ shows that the phase, however, contains useful

7 time delay information for most of the frequency range of interest, from around $25 \mathrm{~Hz}-400 \mathrm{~Hz}$.

8 That the phase data is valid throughout this frequency range is further confirmed with

9 reference to figure $8 \mathrm{c}$, which shoes the coherence between both the source and receiver

10 signals and the voltage reference. Indeed, previous work has shown that unwrapped phase

11 data can remain valid even if the coherences are extremely low (Muggleton et al, 2011).

\section{C Experiments to locate buried cast iron service pipes}

13 The test site used for these experiments was at Blagdon, owned by Bristol Water. The 14 Blagdon test site is a leakage test bed comprising a pressurized cast iron mains pipe with a 15 number of service connections of coming off at intervals (Figure 9). All the service pipes 16 have in-built leaks; however, the water supply to these pipes can be turned on or off as 17 required. All the pipes are buried at the standard burial depth of approximately $1.0 \mathrm{~m}$. Here, 18 measurements are made over two of the cast iron service pipes, one filled with water and one 19 empty. Typically the service pipes are $50-75 \mathrm{~mm}$ in diameter.

20 Measurements were made at one cross section over each pipe. For each cross section, seven

21 geophones were used, spaced at $1 \mathrm{~m}$ intervals, as previously. Again seven excitation positions 22 were used. Here the input signal used was a broadband random excitation between $30 \mathrm{~Hz}$ and $231.6 \mathrm{kHz}$, over approximately 1 minute, using a 4kHz sampling rate.

24 Figures 10a,b shows the cross-sectional image for each of the pipes using a wavespeed of $2575 \mathrm{~m} / \mathrm{s}$, that found to be the optimum, using the form of the cross correlation function given in 26 equation (2). Figures $10 \mathrm{c}, \mathrm{d}$ show the same cross-sections using the modified form of the cross 
1 correlation function given in equation (7). Here there is a marked difference, with the images

2 using the modified form of the cross correlation function being vastly superior, and the

3 darkest areas corresponding to the location of the pipe. The Blagdon site houses a pumping

4 station pumping water to much of the area covered by Bristol water so, as such, can be quite

5 noisy at times. Figures $11 \mathrm{a}, \mathrm{b}$ shows the magnitude and phase of the integrands of equations

6 (2) and (7) respectively for an example source/receiver pairing for the water-filled pipe.

7 Examination of the figures reveal that the effect of using an additional voltage reference in the

8 integrand of equation (7) is two-fold: firstly, at frequencies below approximately $30 \mathrm{~Hz}$, the

9 signal is dominated by environmental noise, most probably originating at the pumping station,

10 which is removed by using the voltage reference; secondly, spikes in the magnitude data at

11 higher frequencies (most notably at $771 \mathrm{~Hz}, 812 \mathrm{~Hz}$ and harmonics thereof) are removed by

12 using the voltage reference. This second phenomenon is the cause of the banded effect seen in

13 Figures 10a,b.

\section{IV. DISCUSSION \& CONCLUSIONS}

15 The results presented here demonstrate that the shear wave ground vibration technique can be 16 used to successfully locate both plastic and cast iron pipes. For the technique to be effective 17 there needs to be a difference between the elastic properties of the object to be detected and 18 those of the surrounding soil. For metal pipes, significant differences would be expected but 19 for plastic pipes (particularly those filled with water) the contrast will not be that great. That the technique is effective here for two of the three polyethylene pipe measurement lines is

21 very encouraging and testament to the robustness of the approach. Moreover, the ability to 22 detect service pipes is of great importance. For the Mapping the Underworld programme, the 23 fact that no access to the pipe is required greatly improves the viability of using vibro24 acoustic techniques in the detection armoury.

25 The smoothed coherence transform has been shown to be effective in increasing the useable 26 frequency range and hence in improving the resolution of the method. Testing on different 
1 sites enabled the cross-correlation formulation given in equation (7) to be evaluated. From

2 these experiments, the indications are that the modified formulation can significantly improve

3 the quality of the final image when operating in a noisy environment.

4 It is evident that much further work is required before this technique can be deployed in an 5 urban environment as part of a multi-sensor device. However, a few areas of current 6 endeavour are particularly worthy of note at this time:

- Within MTU, research into the intelligent analysis and classification of data such as that presented here is well underway as is work to combine the data with that obtained from other sources (Chen and Cohn, 2010,2011 and Dutta et. al, 2013).

- It is evident from the results that wavespeeds in the ground can vary considerably, even over a distance of only a few metres. Although in-situ wavespeed measurements were carried out, the optimum wavespeed used in the stacking calculations was, in this case, finally determined with reference to the "ground truth". Better alternatives are needed and on-going work is addressing the issue of how to identify the most appropriate wavespeed without recourse to burial records which, indeed, frequently may not be available. Improved methods to accurately estimate the wavespeed in-situ, with the inclusion of variations of the wavespeed with depth, are being developed. Furthermore, combining data from other technologies will mitigate some of the uncertainties associated with the wavespeed.

- The technique described in this paper is, in essence, a two-dimensional one. In practice, outside of a controlled test environment, it is possible that the pipes to be detected will not lie perpendicular to any given measurement plane (although, for the most part, they tend to run parallel or perpendicular to road or pavement edges). However, provided that the pipe crosses the measurement plane, then that part of the pipe can potentially be detected. Several appropriately spaced measurement planes would then, of course, be needed to determine the overall pipe run. This relates 


\section{ACKNOWLEDGMENTS} sites and providing on-site assistance.

\section{REFERENCES} Research Needs.

closely to the issue of false targets such as rocks or debris in the soil. For the most part, pipes can be considered to be linear features in the ground so, whilst such unwanted targets may indeed be detected in one measurement plane using this method, it is unlikely that they will appear linearly across a number of planes. In this regard, tree roots may be more problematic; however, the elastic properties of the roots are likely to match better with those of the parent soil than either a plastic or metal pipe, so are less likely to be detected in the first place. That MTU aims to develop a multi-sensor device is of particular importance here. In the final analysis, evidence will be gleaned from all the sensors present and combined intelligently with available maps; this should minimize the problems with identifying false targets.

The authors gratefully acknowledge support provided by the Engineering and Physical Science Research Council (EPSRC) through the EPSRC Grant EP/F065973. The authors are also indebted to both South Staffs water and Bristol water for permitting access to their test

Ashdown C. (2000). Mains Location Equipment - A State of the Art Review and Future UKWIR Report 01/WM/06/1, www.ukwir.org/publicstream.asp?file=80679.pdf(last viewed 24/10/13)

Bendat and Piersol, (1993). Engineering Applications of Correlation and Spectral Analysis. Pp 47-50, John Wiley \& Sons, Inc., London.Carter, G.C., Nuttall, A.H. and Cable, P.G. (1973). The Smoothed Coherence Transform. Proceedings of the IEEE 61, No. 10, 2.

Chen, H. and Cohn, A.G. (2010). Buried Utility Pipeline Mapping based on Street Survey and Ground Penetrating Radar. Proceedings of the 19th European Conference on Artificial Intelligence, Lisbon, Portugal, August 16-20, 2010, 01/2010 
1 Chen, H. and Cohn, A.G. (2011). Buried Utility Pipeline Mapping Based on Multiple Spatial

2 Data Sources: A Bayesian Data Fusion Approach. Proceedings of the 22nd International

3 Joint Conference on Artificial Intelligence, Barcelona, Catalonia, Spain, July 16-22, 2011;

$4 \quad 01 / 2011$

5 Dutta, R., Cohn, A.G. and Muggleton, J.M. (2013). 3D mapping of buried underworld

6 infrastructure using dynamic Bayesian network based multi-sensory image data fusion.

7 Journal of Applied Geophysics $928-19$

8 Gao, Y., Brennan, M.J. and Joseph, P.F. (2006) A comparison of time delay estimators for the

9 detection of leak noise signals in plastic water distribution pipes. Journal of Sound and

10 Vibration 292, No 3-5, 552-70.

11 Knapp, C.H. and Carter, G.C. (1976). The generalised correlation method for estimation of 12 time delay. IEEE Transactions on Acoustics, Speech and Signal Processing24, No. 4, 320-7.

13 Linford, N. (2006) The Application of Geophysical Methods to Archaeological Prospection.

14 Reports on Progress in Physics. 69, No. 7, 2205-2257.

15 McMahon, W., Burtwell, M.H. and Evans, M. (2005). Minimising Street Works Disruption:

16 The Real Costs of Street Works to the Utility Industry and Society. UK Water Industry

17 Research, London, UK. UKWIR Report 05/WM/12/8.

18 Miller, R.D. and Xia, J. (1998). Large Near-Surface Velocity Gradients on Shallow Seismic

19 Reflection Data. Geophysics 63, No. 4, 1348-1356.

20 Muggleton, J.M., Brennan, M.J. (2006). The use of acoustic methods to detect \&

21 locate underground piping systems. Proceedings of the IX International Conference on Recent Advances in Structural Dynamics Southampton, UK. 
1 Muggleton, J.M., Brennan, M.J. (2008). The design and instrumentation of an experimental

2 rig to investigate acoustic methods for the detection and location of underground piping

3 systems. Applied Acoustics 69, 1101-1107.

4 Muggleton, J.M., Brennan, M.J. and Gao, Y. (2011). Determining the location of

5 underground plastic water pipes from measurements of ground vibration. Journal of Applied

6 Geophysics, 75, 54-61.

7 Papandreou, B.D. (2011) On the Detection of Shallow Buried Objects Using Seismic Wave 8 Reflections. PhD Thesis, Institute of Sound and Vibration Research, The University of

9 Southampton.

10 Papandreou, B.D., Brennan, M.J. and Rustghi, E. (2011). On the Detection of Objects Buried 11 at a Shallow Depth Using Seismic Wave Reflections. Journal of the Acoustical Society of 12 America 129, No. 3, pp1366-1374.

13 Papandreou, B.D., Rustighi, E., Brennan, M.J. (2009). On the detection of shallow buried 14 objects using seismic wave reflections. Proceedings of the XVI International Congress on 15 Sound and Vibration, Krakov, Poland.

16 Royal, A.C.D., Rogers C.D.F, Atkins P.R., Brennan, M.J., Chapman, D.N., Cohn, A.G., 17 Curioni, G., Foo, K.Y, Goddard, K., Lewin, P.L., Metje, N., Muggleton, J.M., Naji, A., 18 Pennock, S.R., Redfern, M.A., Saul, A.J., Swingler, S.G. and Wang, P. (2010). Mapping the 19 Underworld Phase II - Latest Developments. International No-Dig 2010. 28th International 20 Conference on the Trenchless Installation of Utilities, Singapore, 8-10 November.

21 Royal, A.C.D., P. Atkins, M.J. Brennan, D.N. Chapman, H. Chen, A.G. Cohn, K.Y. Foo, K.F. 22 Foddard, R. Hayes, T. Hao, P.L. Lewin, N. Metje, J.M. Muggleton, A. Naji, G. Orlando, S.R. 23 Pennock, M.A. Redfern, A.J. Saul, S.G. Swingler, P. Wang, and C.D.F. Rogers. (2011). Site 24 Assessment of Mutliple-Sensor Approaches for Buried Utility Detection. International 
1 Journal of Geophysics, Article ID496123, 19 pages, http://dx.doi.org/10.1155/2011/496123

2 (last viewed 24/10/13)

3 Sabatier, J. and Gilbert, K. (2000). Method for Detecting Buried Objects By Measuring

4 Seismic Vibrations Induced by Acoustical Coupling with a Remote Source of Sound. U.S.P.

5 Office, Editor: United States.

6 Schnieder, W.A. (1984). The Common Depth Point Stack. Proceedings of the IEEE, 72, No.

$7 \quad 10,1238-1256$.

8 Scott, W.R., Jr., Schroeder, C.T., Martin, J.S. and Larson G.D. (2001) Use of Elastic Waves

9 for the Detection of Buried Land Mines. IEE Proc 3, 1116-1118

10 Shin and Hammond. (2008). Fundamentals of Signal Processing for Sound and Vibration

11 Engineers. John Wiley \& Sons, Inc., London, pp90-93.

12 Sugimoto, T., Saitou, H. and Okujima, M. (2000). Study of Underground Imaging Using

13 Shear Waves: the Stacking Method of the Reflected Scattered Wave. Archaeological 14 Prospection 7, 249-261.

15 White, P. (1969). Cross Correlation in Structural Systems: Dispersion and Nondispersion 16 Waves. Journal of the Acoustical Society of America 45. No. 5, 1118-1128. 


\section{$1 \quad$ Figure captions}

2 Figure 1 - Diagram of the experimental setup used for the imaging method. The circles on the

3 surface denote geophones and the square on the surface the source.

4 Figure 2 - Schematic of the coupling platform, used for horizontal excitation. Dimensions are 5 given in millimetres. The top platform structure was made of aluminium with the prongs 6 made of steel.

7 Figure 3. Arrangement of shaker and geophones for ground excitation measurements using

8 shear waves

9 Figure 4. Velocity time histories in three orthogonal directions. At source location (in this 10 case location 1); (b) At receiver location (in this case at location 7, 6m from the source)

11 Figure 5. Cross-sectional stacking images for the first measurement line using 6 different 12 wavespeeds

13 Figure 6. Cross-sectional stacking images for the second measurement line using 2 different 14 wavespeeds

15 Figure 7. Cross-sectional stacking images for the first measurement line using 6 different 16 wavespeeds without pre-whitening (SCOT)

17 Figure 8. Modified cross spectra, as used in the integrands of equation (7), source at location

18 1, receiver at location 2. (a) magnitude; (b) phase; (c) coherence.

19 Figure 9. Test site at Blagdon

20 (here the run of the main pipe and those of three service pipes can be seen on the ground 21 surface)

22 Figure 10. Cross sectional stacking image at single wavespeed; the arrow indicates the lateral 23 position of the pipe as determined from inspection at the ground surface. (a) water filled pipe 24 (using equation 7)); (b) empty pipe (using equation 7); (c) water filled pipe (using equation $25 \quad$ 2); (d) empty pipe (using equation 2)

26 Figure 11. Modified cross spectra, as used in the integrands of equations (2) and (7), source at 27 location 1, receiver at location 2. (a) magnitude; (b) phase. Note the different frequency scales 


\section{Figures}

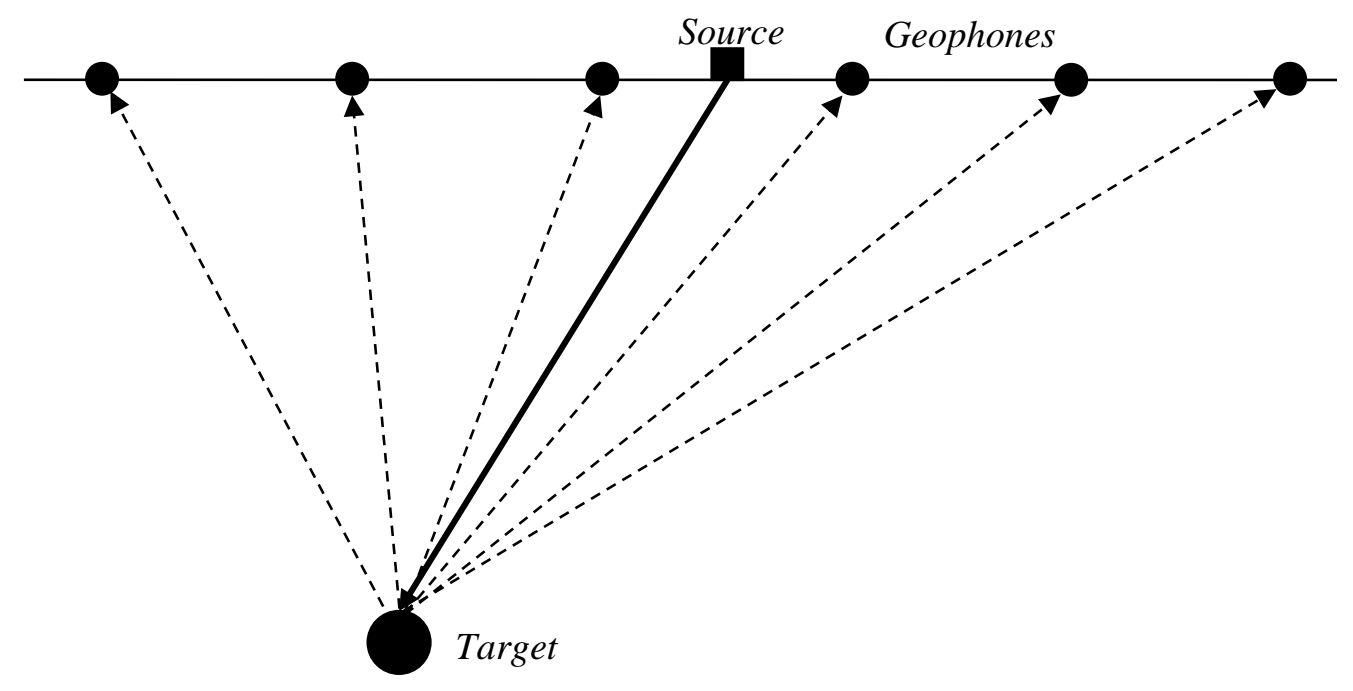

2

3 Figure 1 - Diagram of the experimental setup used for the imaging method. The circles on the surface 4 denote geophones and the square on the surface the source. 

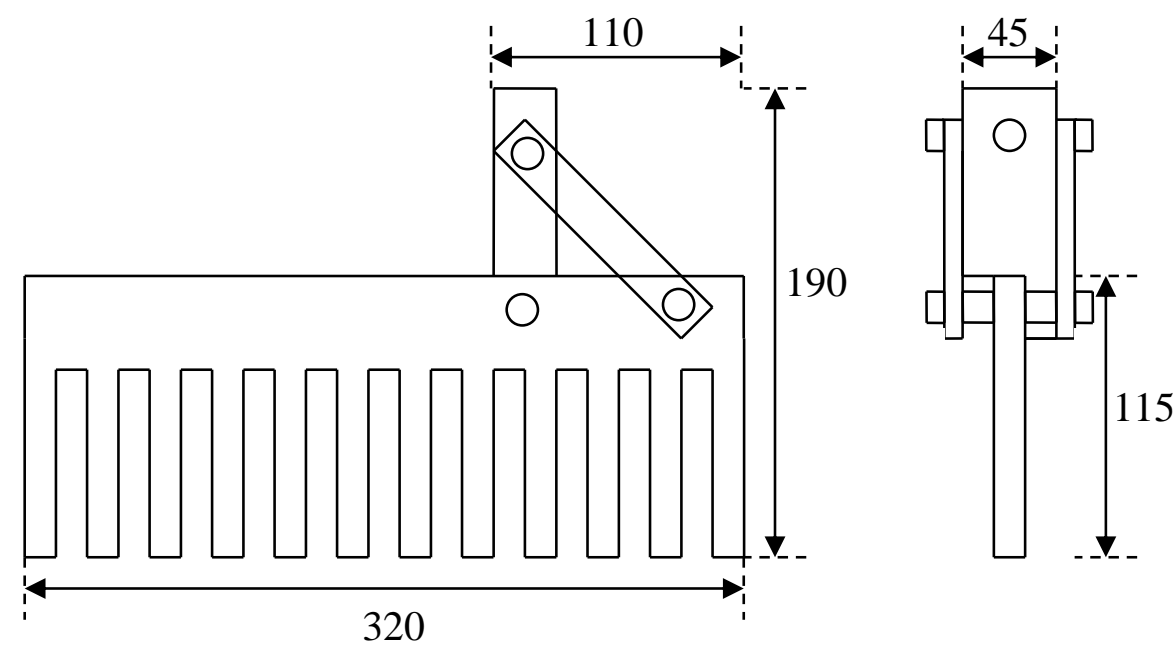

3

4 Figure 2 - Schematic of the coupling platform, used for horizontal excitation. Dimensions are given in 5 millimetres. The top platform structure was made of aluminium with the prongs made of steel.

6 
shaker with coupling platform

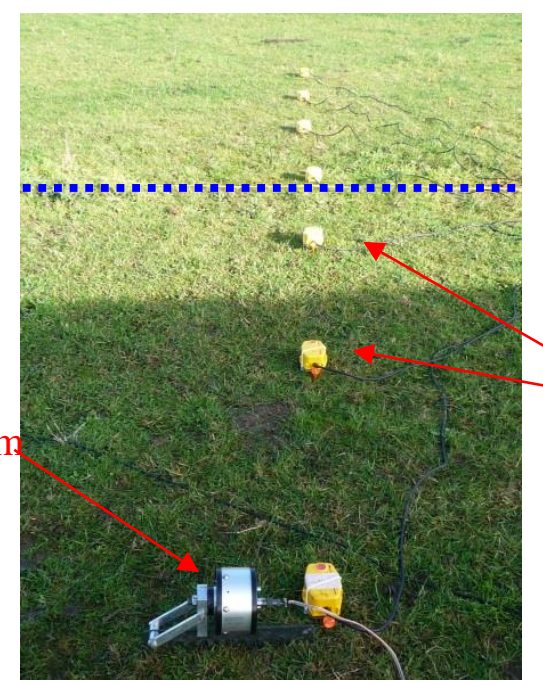

approximate run of pipe

Figure 3. Arrangement of shaker and geophones for ground excitation measurements using shear waves 

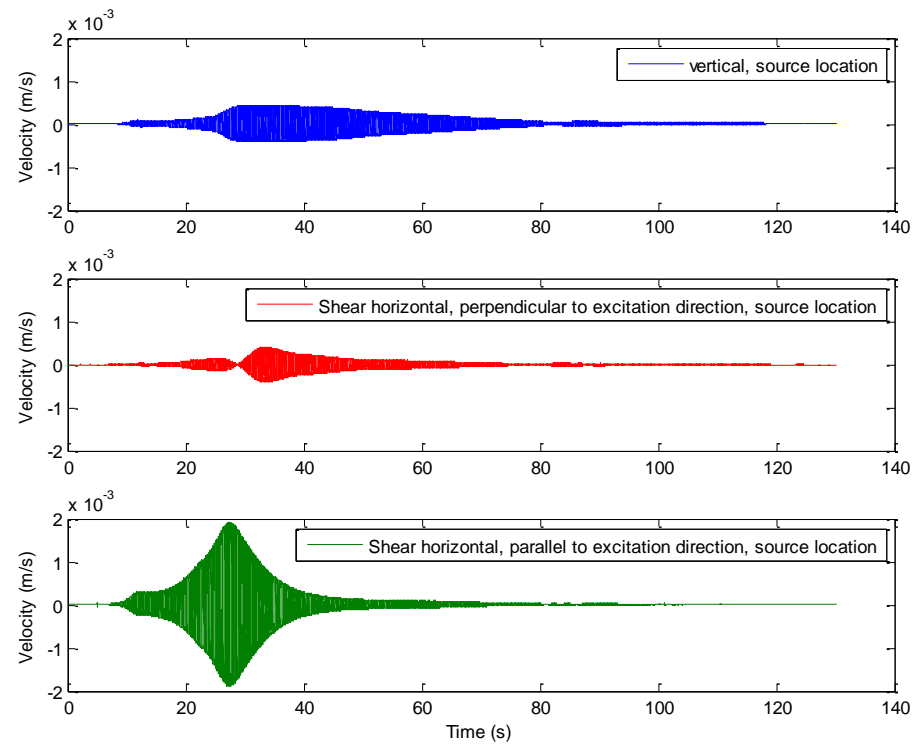

(a)
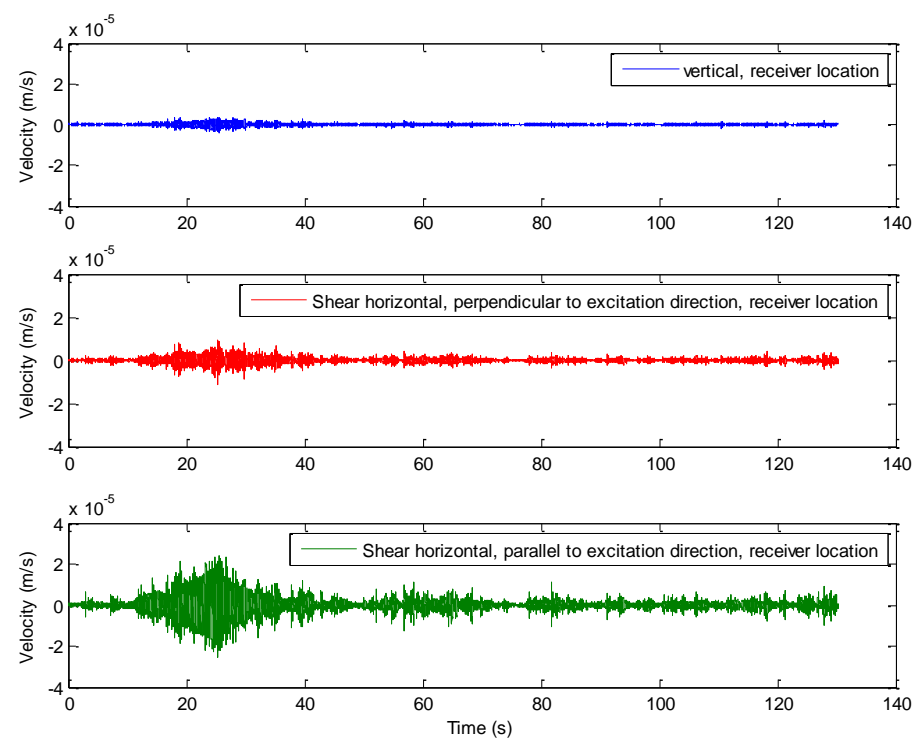

3
4

5

6

7

8

9

(b)

\section{Figure 4}

Velocity time histories in three orthogonal directions

(a) At source location (in this case location 1); (b) At receiver location (in this case at location $7,6 \mathrm{~m}$ from the source) 
3
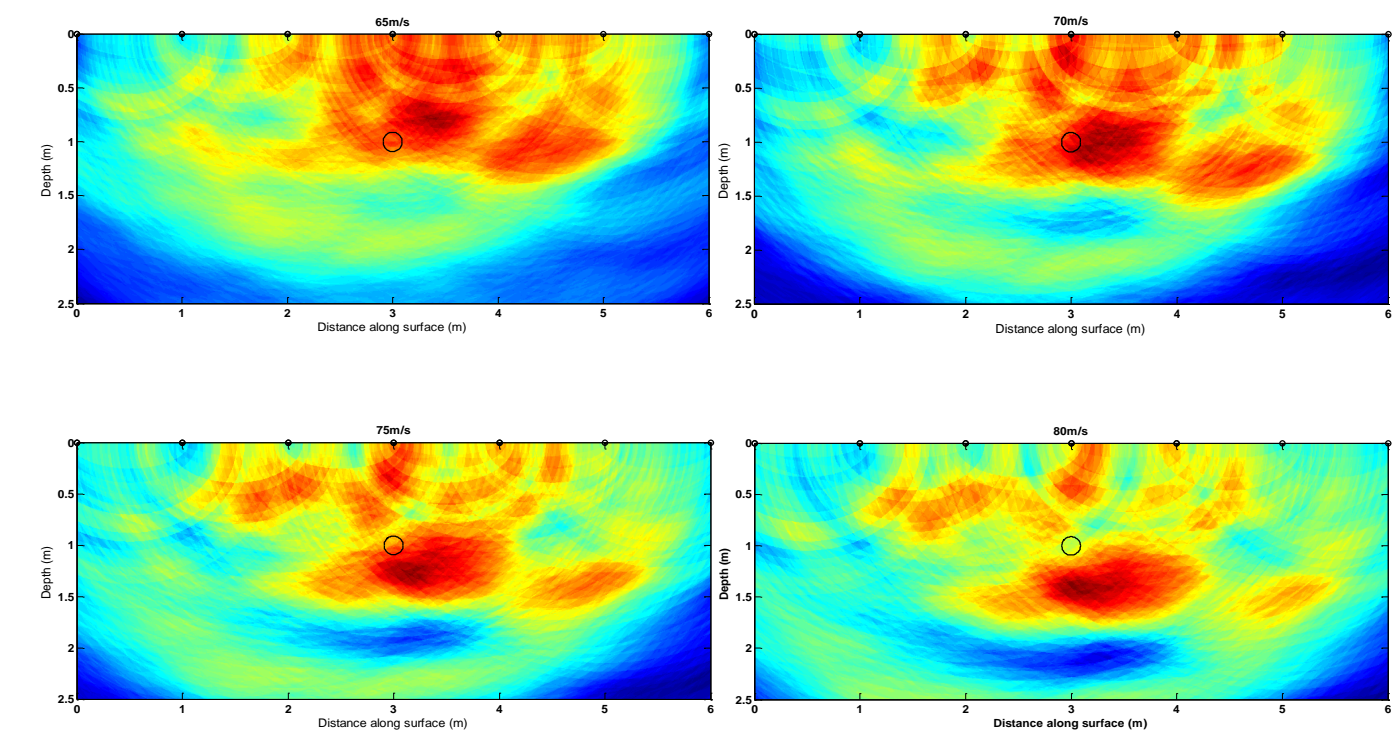

4
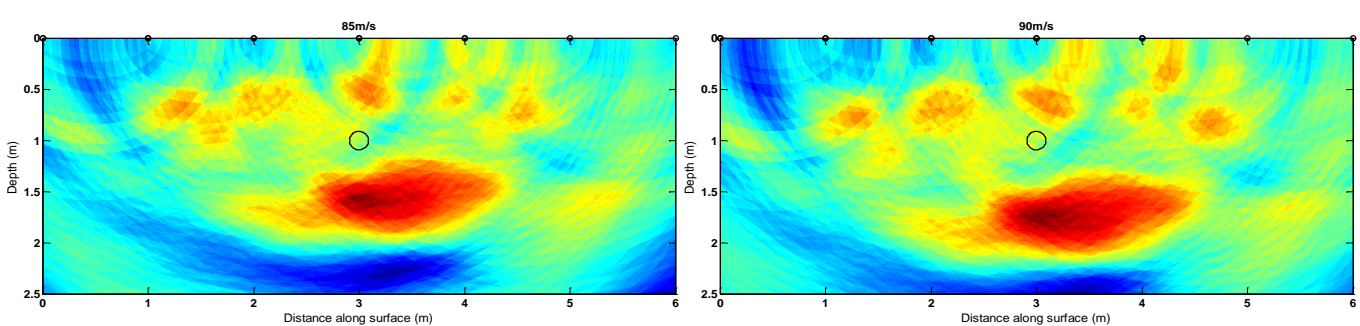

5

6 Figure 5. Cross-sectional stacking images for the first measurement line using 6 7 different wavespeeds

8

9 

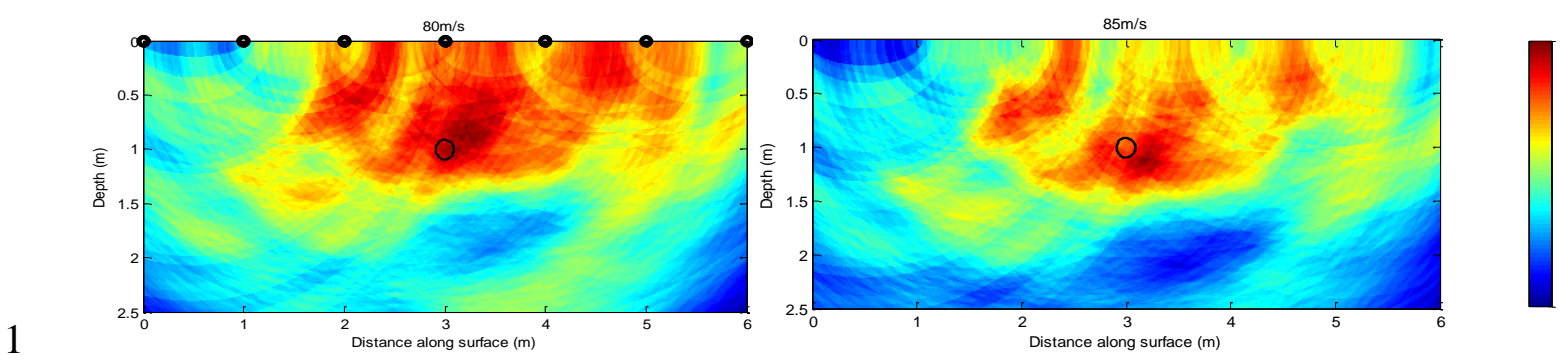

2 Figure 6. Cross-sectional stacking images for the second measurement line using 2 3 different wavespeeds

4
5 

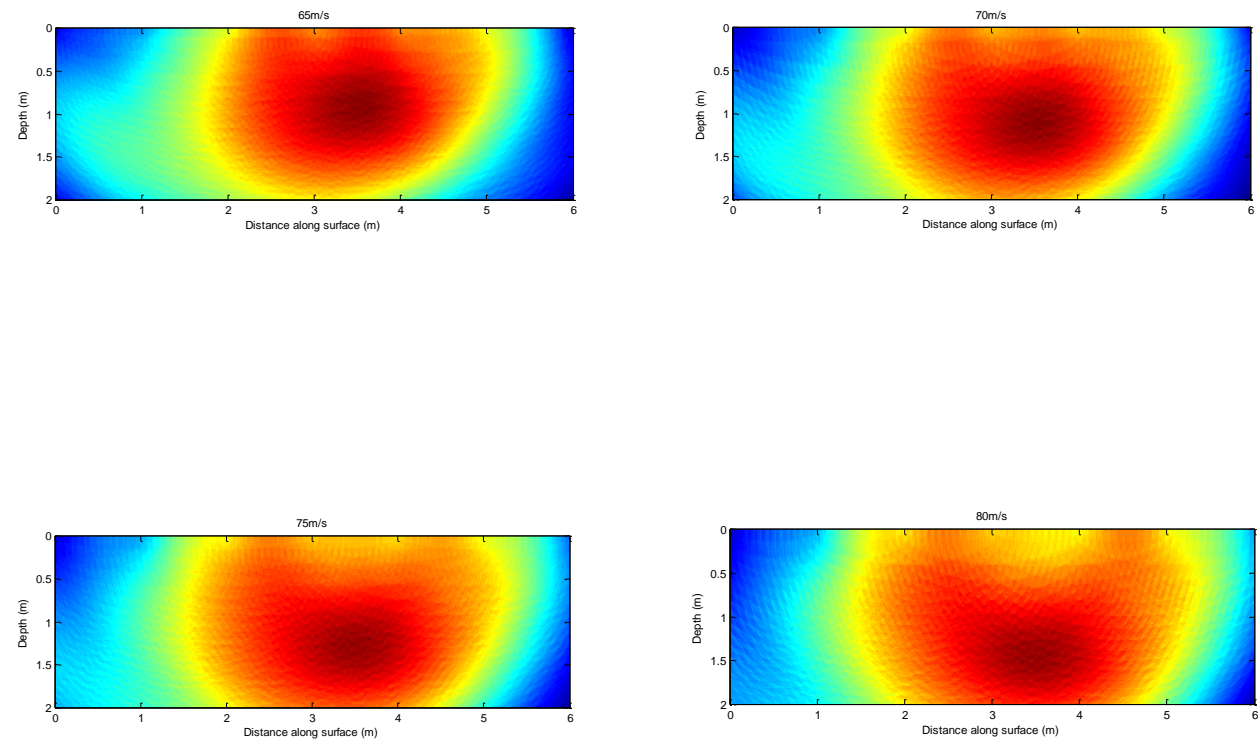

2
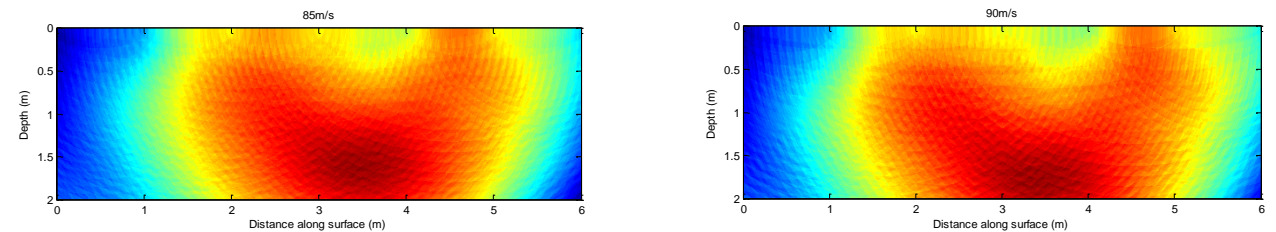

3

4 Figure 7. Cross-sectional stacking images for the first measurement line using 6 5 different wavespeeds without pre-whitening (SCOT)

6

7

8 


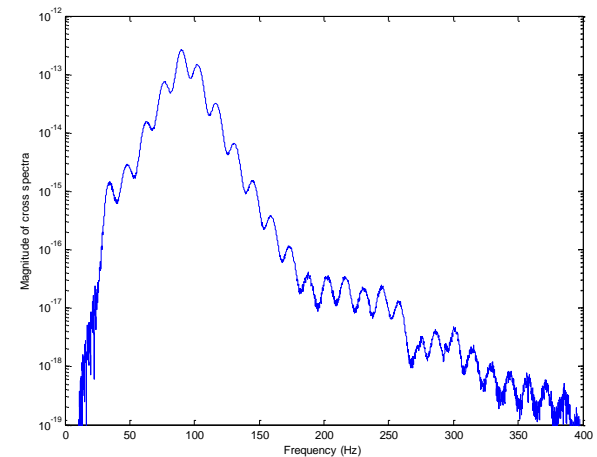

(a)

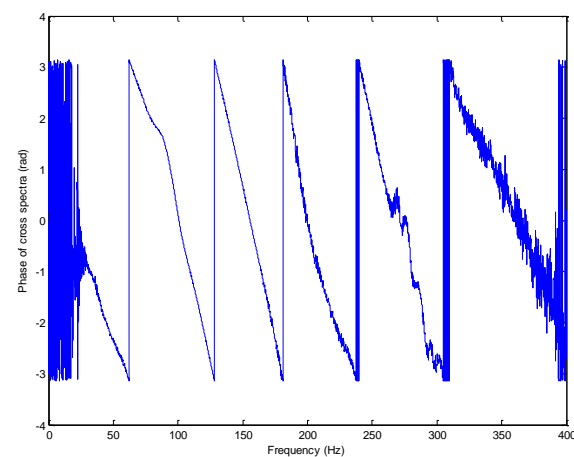

(b)
3

4

5

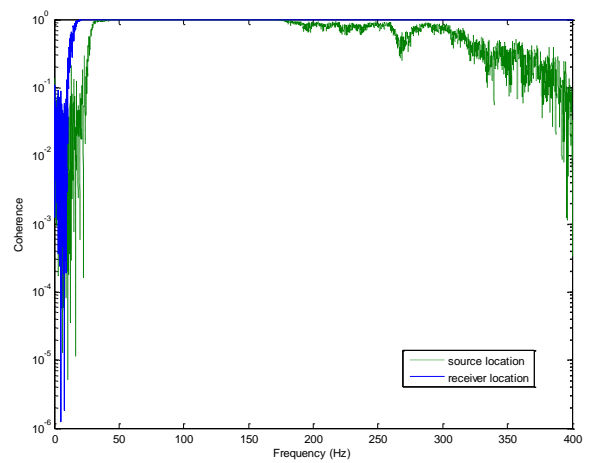

(c)

Figure 8

6 Modified cross spectra, as used in the integrands of equation (7), source at location 1, receiver at location 2. (a) magnitude; (b) phase; (c) coherence. 


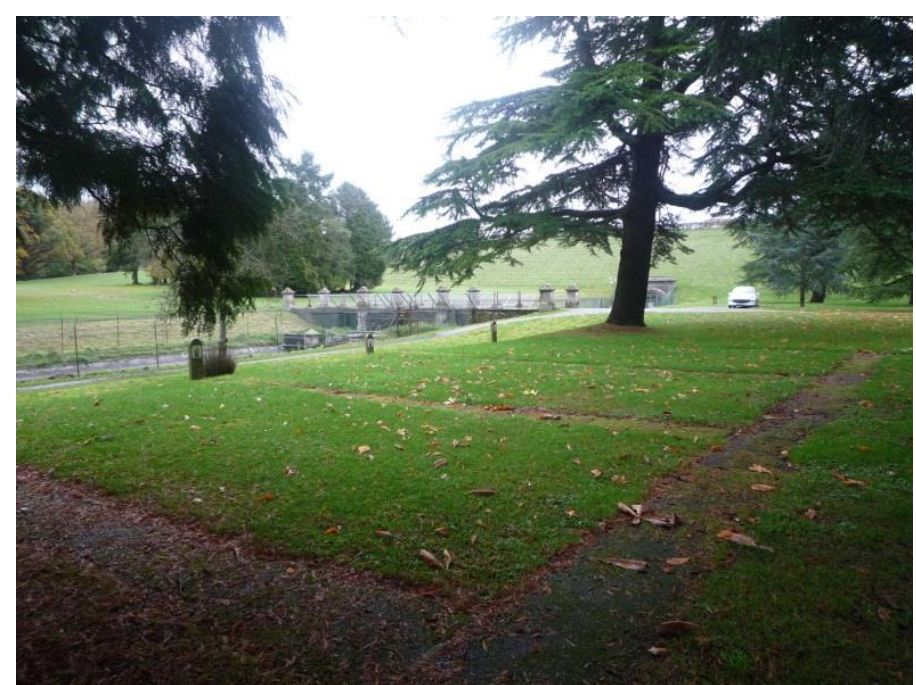

3 Figure 9

4 Test site at Blagdon

5 (here the run of the main pipe and those of three service pipes can be seen on the 6 ground surface) 


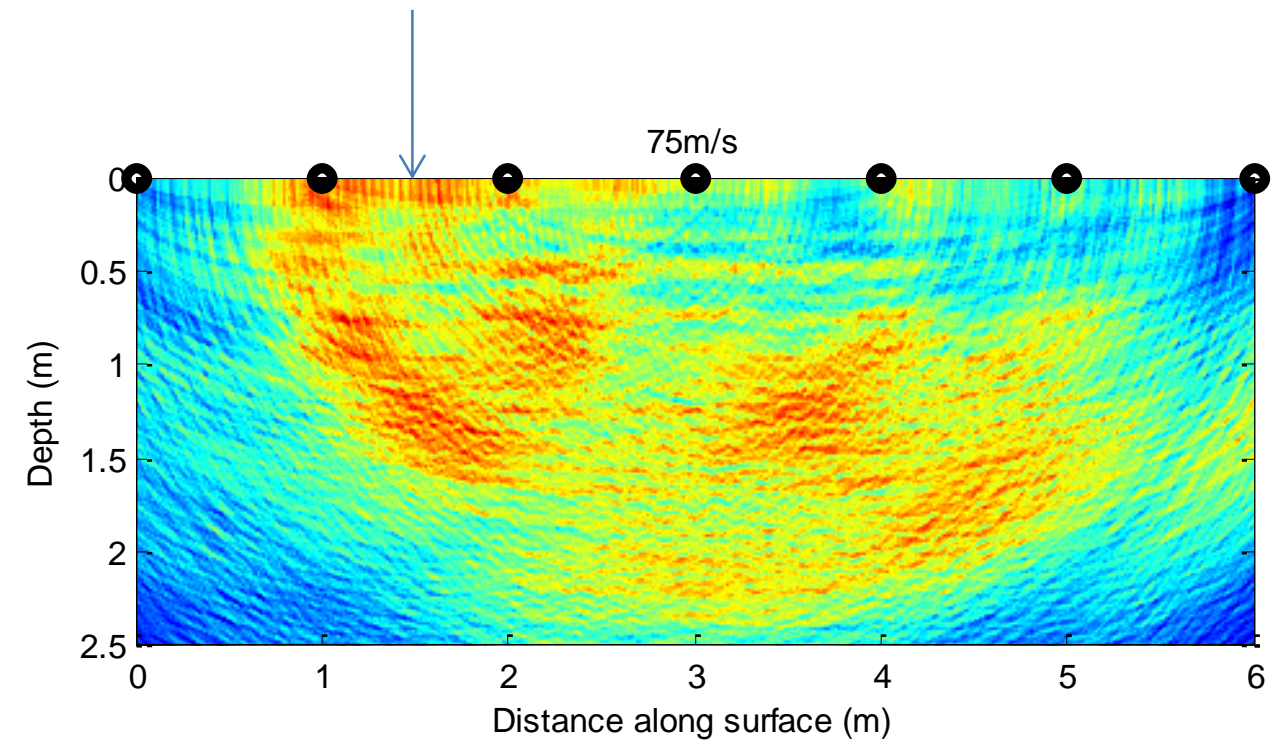




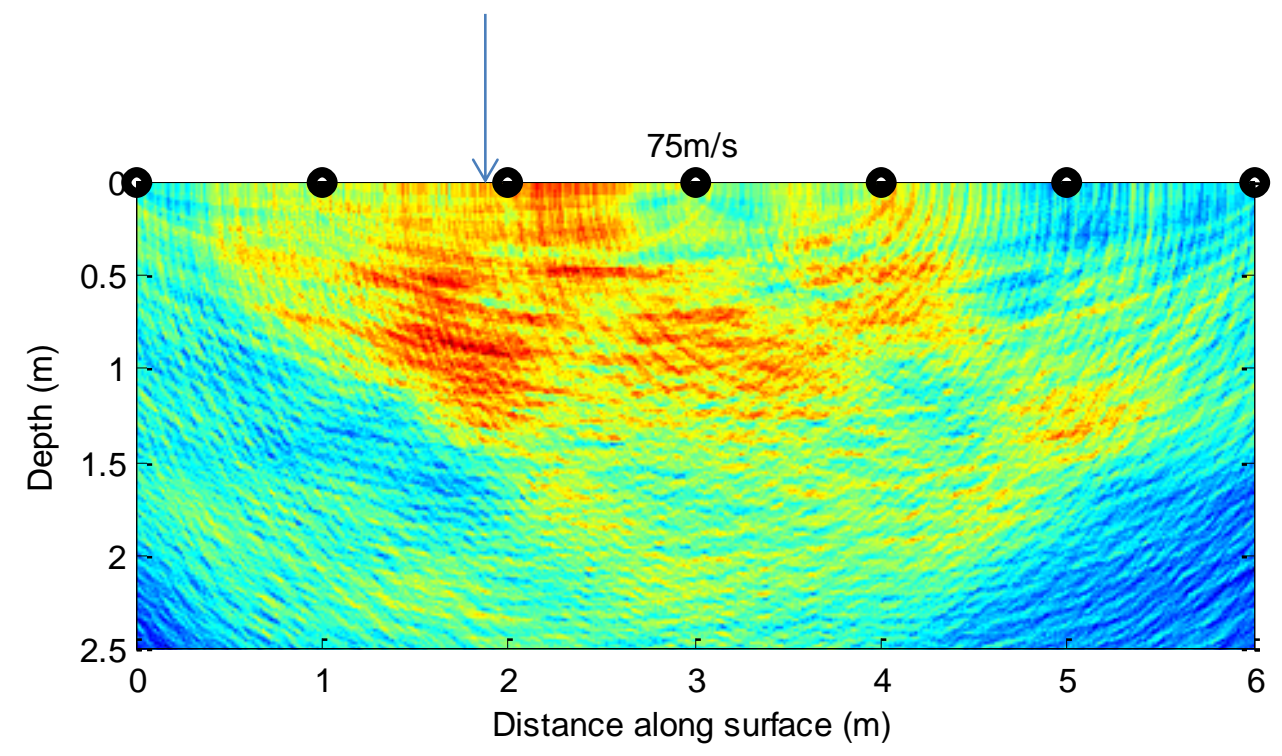




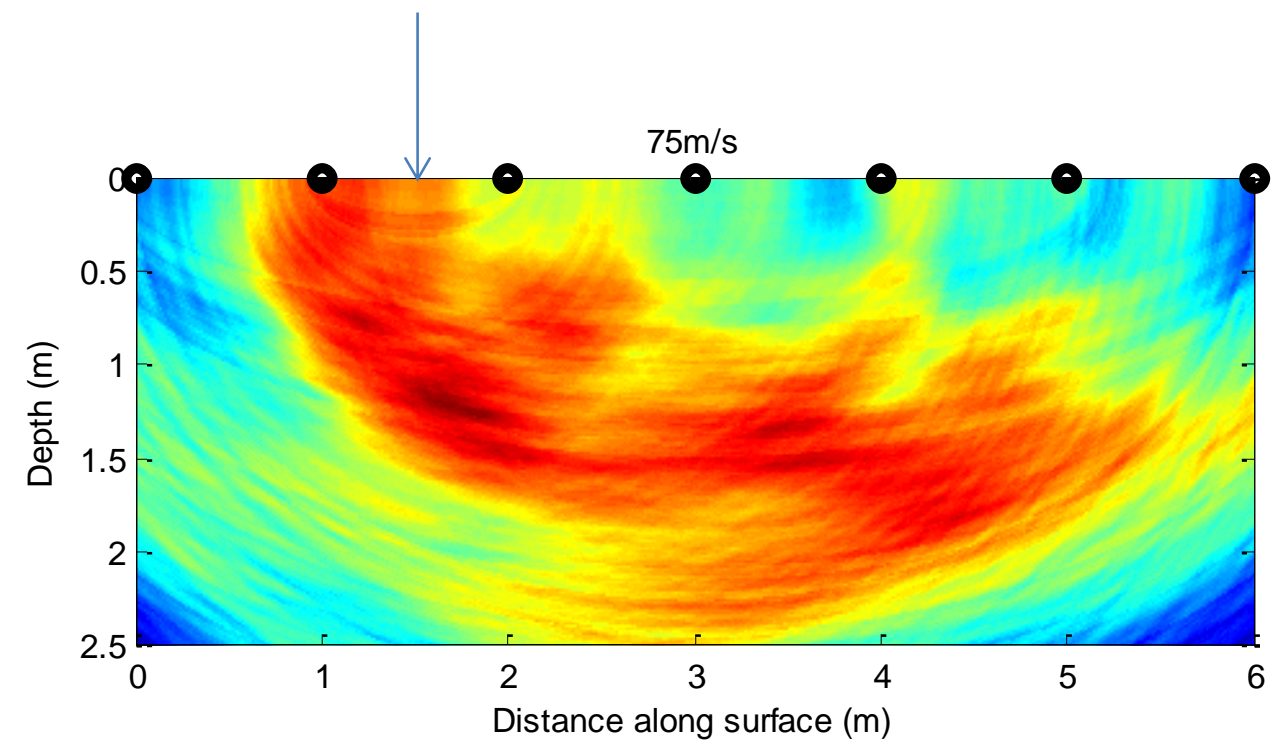




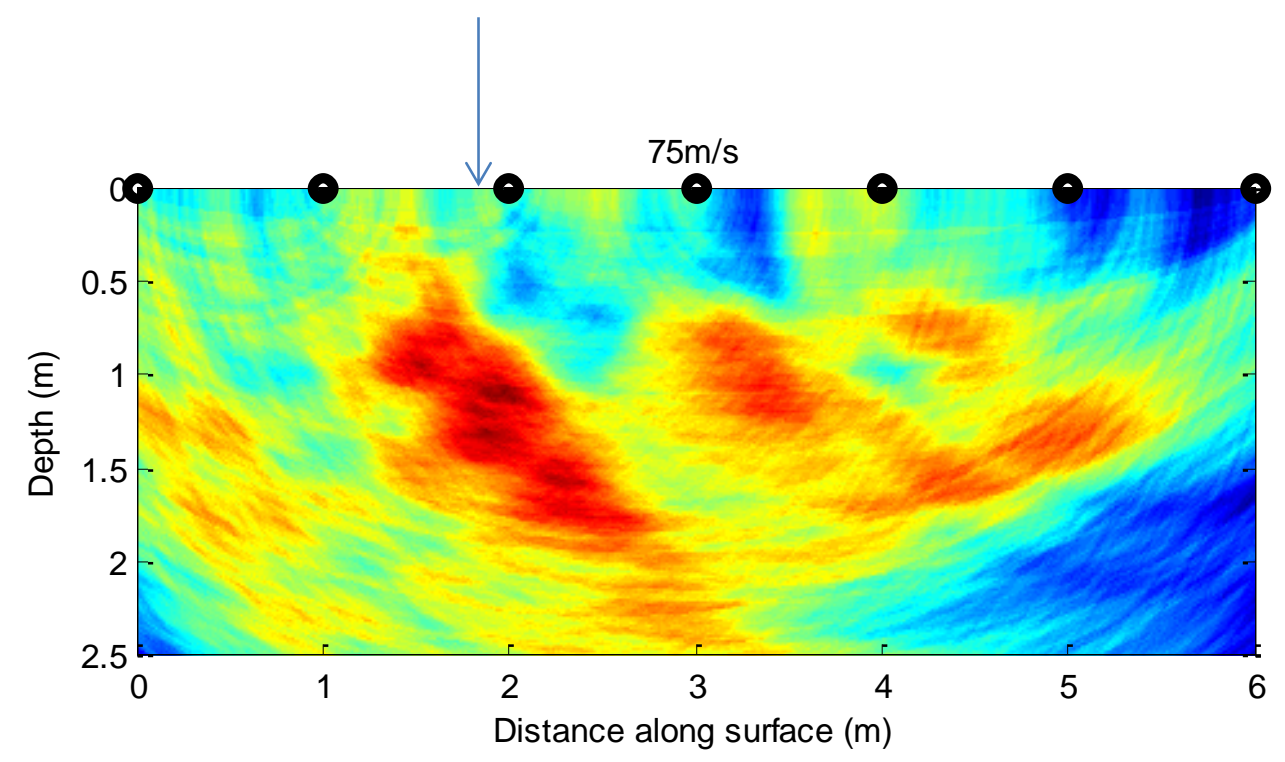

Figure 10

4 Cross sectional stacking image at single wavespeed; the arrow indicates the lateral position of

5 the pipe as determined from inspection at the ground surface. (a) water filled pipe (using

6 equation 2)); (b) empty pipe (using equation 2); (c) water filled pipe (using equation 7); (d) 


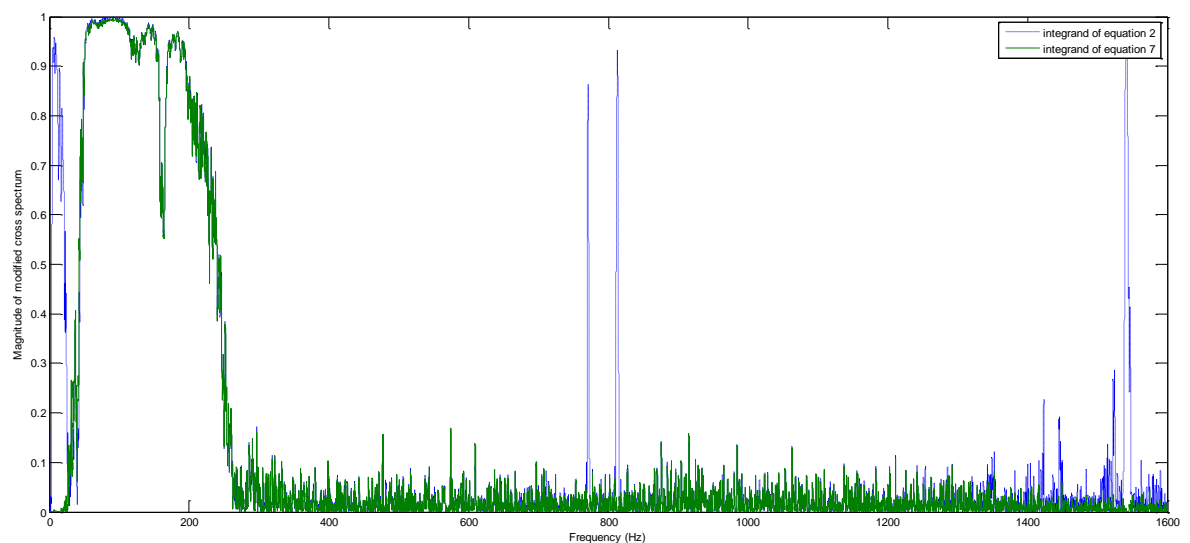

2

3

(a)

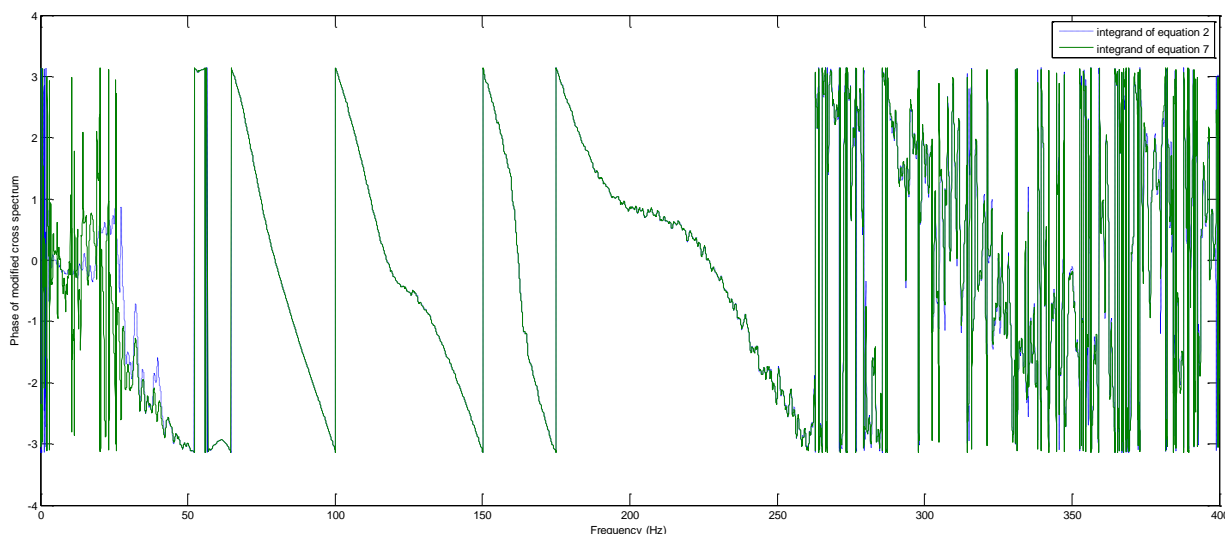

4

5

6

Figure 11

7 Modified cross spectra, as used in the integrands of equations (2) and (7), source at location 1,

8 receiver at location 2. (a) magnitude; (b) phase. Note the different frequency scales

9 